\title{
Integrin expression in stem cells from bone marrow and adipose tissue during chondrogenic differentiation
}

\author{
ULRICH REINHART GOESSLER $^{1}$, PETER BUGERT $^{2}$, KAREN BIEBACK ${ }^{2}$, \\ JENS STERN-STRAETER ${ }^{1}$, GREGOR BRAN ${ }^{1}$, KARL HÖRMANN ${ }^{1}$ and FRANK RIEDEL ${ }^{1}$
}

\begin{abstract}
${ }^{1}$ Department of Otolaryngology, Head and Neck Surgery, University Hospital Mannheim, University of Heidelberg, Mannheim; ${ }^{2}$ Institute of Transfusion Medicine and Immunology, Red Cross Blood Service of Baden-Württemberg/ Hessen, Faculty of Clinical Medicine, Ruprecht Karls University of Heidelberg, Mannheim, Germany
\end{abstract}

Received October 3, 2007; Accepted November 29, 2007

\begin{abstract}
The use of adult mesenchymal stem cells (MSC) in cartilage tissue engineering offers new perspectives in the generation of transplants for reconstructive surgery. The extracelular matrix (ECM) plays a key role in modulating the function and phenotype of the embedded cells and contains the integrins as adhesion receptors mediating cell-cell and cell-matrix interactions. In our study, characteristic changes in integrin expression during the course of chondrogenic differentiation of MSC from bone marrow and adipose tissue were compared. MSC were isolated from bone marrow biopsies and adipose tissue. During cell culture, chondrogenic differentiation was performed. The expression of integrins and their signaling components were analysed with microarray and immunohistochemistry in freshly isolated MSC and after chondrogenic differentiation. The fibronectin receptor (integrin $\alpha 5 \beta 1$ ) was expressed by undifferentiated MSC, and expression rose during chondrogenic differentiation in both types of MSC. The components of the vitronectin/osteopontin receptors $(\alpha \mathrm{v} \beta 5)$ were not expressed by freshly isolated MSC, and expression rose with ongoing differentiation. Receptors for the collagens $(\alpha 1 \beta 1, \alpha 2 \beta 1, \alpha 3 \beta 1)$ were weakly expressed by undifferentiated MSC and were activated during differentiation. Intracellular signaling components integrinlinked kinase (ILK) and CD47 showed increased expression with ongoing differentiation. For all integrins, no significant differences were be found in the 2 types of MSC. Integrinmediated signaling appeared to play an important role in the generation and maintenance of the chondrocytic phenotype
\end{abstract}

Correspondence to: Dr Ulrich Goessler, Department of Otolaryngology, Head and Neck Surgery, University Hospital Mannheim, D-68135 Mannheim, Germany

E-mail: ulrich.goessler@hno.ma.uni-heidelberg.de

Key words: integrin, cartilage, tissue engineering, differentiation, extracellular matrix, mesenchymal stem cells, chondrogenic differentiation during chondrogenic differentiation. Particularly, the receptors for fibronectin, vitronectin, osteopontin and the collagens may be involved in the generation of the ECM. Intracellularly, their signals might be transduced by ILK and CD47. To fully harness the potential of these cells, future studies should be directed to ascertain their cellular and molecular characteristics for optimal identification, isolation, and expansion.

\section{Introduction}

Each year millions suffer from organ failure or tissue loss due to injury, disease, or congenital malformation $(1,2)$. With a progressively aging population, there is an increasing demand for therapies to regenerate or replace musculoskeletal tissues. More than three million musculoskeletal procedures are performed annually. The existing shortage of donor tissue and organs available for transplantation has driven a multidisciplinary effort to develop therapeutic solutions.

The emerging field of tissue engineering promises to deliver improvements in the technologies and therapies for musculoskeletal disorders through the development of biological substitutes for tissue replacement. The creation of functional tissue addresses very complex biological problems, comprising a wide range of engineering, science and clinical disciplines. The next generation of engineered musculoskeletal tissues will be more complex and structurally organized to better mimic normal tissue structure and function. Tissue engineering as an interdisciplinary approach utilizes specific combinations of cells, scaffolds and bioactive factors to create, influence and maintain cellular phenotype and function (3).

Cartilage is a unique avascular, aneural and alymphatic loadbearing living tissue. It is unique in that the extracellular matrix is composed of a complex combination of type II collagen fibrils which are specifically arranged and are bonded to very large water-retaining molecules called aggrecan molecules (4). The promise of tissue engineering is perhaps most relevant to chondrogenic defects because cartilage has little self-healing potential.

The successful isolation of human stem cells from bone marrow, periosteum and fat tissue was established by 
different groups (5-8). These cells are highly proliferative and are capable of differentiating into different types of tissue such as bone, cartilage, tendon, muscle or fat. Human mesenchymal stem cells are characterized by a specific pattern of cell surface markers, growth factors, cytokine receptors, integrins and other adhesion molecules $(9,10)$.

Although bone marrow (BM) has been the main source for the isolation of multipotent MSC and BM-MSC are well characterized and safe for handling, the harvesting of BM is a highly invasive procedure and the number, differentiation potential, and maximal life span of MSC from BM decline with increasing age (11-13). Therefore, alternative sources from which to isolate MSC are subject to intensive investigation. Adipose tissue (AT) is an alternative source that can be obtained by a less invasive method and in larger quantities than BM. It has been demonstrated that AT contains stem cells similar to BM-MSC, which are termed processed lipoaspirate (PLA) cells, and these cells can be isolated from cosmetic liposuction in large numbers and grown easily under standard tissue culture conditions $(14,15)$. The multilineage differentiation capacity of PLA cells has been proven in previous studies $(14,15)$.

As cellular function and phenotype are influenced by intrinsic and extrinsic stimuli, the cell-cell and cell-matrix interactions are of special interest in understanding factors crucial to generation of a distinct cellular phenotype. The integrin family of cell surface receptors appears to play a major role in the mediation of the cell-ECM interactions associated with structural and functional changes in surrounding tissues (16-19). The integrins are heterodimeric glycoproteins that are composed of an $\alpha$ - and a $\beta$-subunit, each of which has extracellular and cytoplasmic domains. The extracellular domains bind to a number of ECM proteins, including collagen types II and VI, fibronectin and matrix Gla protein. Several previous studies have provided evidence that chondrocytes express integrins (20-25). Salter et al used immunohistochemical staining in normal adult articular cartilage, and noted that integrin $\alpha 5 \beta 1$ was the most prominently expressed chondrocyte integrin (24). A subsequent study demonstrated that the chondrocyte expression of $\alpha 1 \beta 1$, $\alpha 5 \beta 1$ and $\alpha v \beta 5$ were accompanied by weak expression of integrin $\alpha 3 \beta 1$ and $\alpha v \beta 3$ (23). Other integrins are known to have distinct functions in binding components of the ECM (Table I).

Integrin-mediated signaling is involved in a variety of cellular processes such as differentiation, adhesion and migration. Hannigan et al found that integrin-linked kinase (ILK) co-immunoprecipitated with $\beta-1$ integrin from cell lysates, and that overexpression of ILK disrupted cell architecture and inhibited adhesion to integrin substrates, suggesting that ILK regulates integrin-mediated signal transduction (26). In addition to ILK, integrin cytoplasmic domain-associated protein 1 (ICAP-1) interacts with the cytoplasmic domain of $\beta-1$ integrin (27). CD47 or integrinassociated protein (IAP) is a membrane protein that is involved in the increase in intracellular calcium concentration that occurs upon cell adhesion to the extracellular matrix (28).

As the stem cell is responsible for modulating its environment and the chondrocyte phenotype is influenced by the diverse components of the extracellular matrix, the investigation of the molecular basis of distinct changes during developmental processes, for the generation of cartilage transplants especially the process of chondrogenic differentiation, might broaden the understanding of impediments in the field of tissue engineering. As BM-MSC are best characterized, we asked whether MSC derived from other sources share the characteristic expression patterns of BMMSC. The aim of our study was to analyse MSC isolated from $\mathrm{BM}$ and AT under identical in vitro conditions and during chondrogenic differentiation with respect to integrin expression.

\section{Materials and methods}

Collection and isolation of MSC from bone marrow (BM). Bone marrow was obtained from the femoral shaft of patients undergoing total hip replacement at the orthopedic department of the University Hospital Mannheim. Cells were aspirated into a 5-ml syringe containing CPD anticoagulant. In total, six specimens from female patients were obtained, with the donor age ranging from 68 to 84 years.

To isolate mononuclear cells (MNC), the bone marrow aspirates were diluted 1:5 with PBS/2 mM EDTA (Nexell, Baxter, Unterschleissheim, Germany, and Merck, Darmstadt, Germany) and carefully loaded into Ficoll-Hypaque solution (Amersham, Freiburg, Germany). After density gradient centrifugation at $435 \mathrm{x} \mathrm{g}$ for $30 \mathrm{~min}$ at room temperature, MNC were removed from the interphase and washed two to three times with PBS/EDTA. Cell counts were performed using an automated cell analyzer (Cell-Dyn 3200, Abbott, Wiesbaden, Germany).

BM-derived MNC were set in culture at a density of $1 \times 10^{5} / \mathrm{cm}^{2}$ into $75 \mathrm{~cm}^{2}$ tissue culture flasks (Nunc, Wiesbaden, Germany) in MSCGM medium (MSCGM BulletKit ${ }^{\mathrm{TM}}$, Cambrex, St. Katharinen, Germany).

After overnight incubation at $37^{\circ} \mathrm{C}$ in a humidified atmosphere containing $5 \% \mathrm{CO}_{2}$, non-adherent cells were removed and fresh medium was added to the flasks. Cultures were maintained and remaining non-adherent cells were removed by complete exchange of culture medium every three to four days. The flasks were screened continuously to access developing colonies of adherent cells. Fibroblastoid cells were recovered between day 7 and 10 after initial plating by using $0.04 \%$ trypsin/0.03\% EDTA (PromoCell, Heidelberg, Germany). Recovered cells were replated at a density of 4000-5000 cells $/ \mathrm{cm}^{2}$ as passage 1 (P1) cells and thereafter.

Collection of adipose tissue (AT). AT was obtained from 18 donors ranging in age from 26 to 57 years undergoing liposuction procedures. Lipoaspirates were obtained in accordance with the standard of ethics of the local ethics committee.

Isolation and culture of cells from adipose tissue (AT). The raw lipoaspirate $(50-100 \mathrm{ml})$ was processed as described previously by Goessler et al (17). To isolate the stromal vascular fraction (SVF), lipoaspirates were washed vigorously with PBS. Thereafter the lipoaspirates were digested with an equal volume of $0.075 \%$ collagenase type I (Sigma-Aldrich, St. Louis, MO, USA) for $30-60 \mathrm{~min}$ at $37^{\circ} \mathrm{C}$ with gentle 
agitation. The activity of the collagenase was neutralized with DMEM-lg containing 10\% FCS. To obtain the highdensity SVF pellet, the digested lipoaspirate was centrifuged at $1,200 \mathrm{x} \mathrm{g}$ for $10 \mathrm{~min}$. The pellet was re-suspended in MSCGM or DMEM-lg containing 10\% MSCGS and filtered through a 100-fim nylon cell strainer (Falcon). The filtered cells were centrifuged at $1,200 \mathrm{x} \mathrm{g}$ for $10 \mathrm{~min}$. The resuspended SVF cells were plated at a density of $1 \times 10^{6} / \mathrm{cm}^{2}$ into T75 or T175 culture flasks. Nonadherent cells were removed 12-18 $\mathrm{h}$ after the initial plating by vigorously washing the plates. The resulting fibroblastoid adherent cells were termed AT-derived fibroblastoid adherent cells (ATFACs). AT-FACs were cultivated under the same conditions as described for BM-FACs. AT-FACs were harvested at subconfluence using trypsin. Cells at the second passage and thereafter were replated at a mean density of $1.8 \pm 3.1 \times 10^{3} / \mathrm{cm}^{2}$.

The generation of single separated fibroblast colony forming units (CFU-F) was achieved by initially seeding the SVF cells at a low density $\left(1 \times 10^{2}\right.$ to $1 \times 10^{3}$ cells per $\left.\mathrm{cm}^{2}\right)$. CFU-F were selected and isolated as described for BM-CFU-F. Subcultivation of the cells was performed as described for the AT-FACs.

Chondrogenic differentiation. To promote chondrogenic differentiation, $2.5 \times 10^{5}$ cells were gently centrifuged ( $150 \mathrm{x} \mathrm{g}$, $5 \mathrm{~min}$ ) in a $15-\mathrm{ml}$ polypropylene tube (Greiner) to form a pellet according to the protocol of Mackay et al (29). Without disturbing the pellet, the cells were cultured for four weeks in complete chondrogenic differentiation medium (Cambrex) including $10 \mathrm{ng} / \mathrm{ml}$ TGFB3 (Strathmann Biotec AG, Hamburg, Germany) by feeding twice a week. After the culture period, cryosections were analyzed by Safranin O staining. The sections were fixed with ice-cold acetone (Sigma) and stained with $0.1 \%$ aqueous Safranin O solution (Sigma). Cell nuclei were counterstained with Weigert's iron hematoxylin (Sigma).

For the RNA analysis we harvested and lysed the aggregates in RLT buffer (Qiagen). The lysis was aggravated by freezing the pellet repeatedly in liquid nitrogen.

RNA extraction and microarray hybridization. Extraction of RNA was performed using the RNA Mini Kit (Qiagen, Hilden, Germany) according to the manufacturer's protocol and as previously published (30). The RNA concentration was estimated from the absorbance at $260 \mathrm{~nm}$.

Approximately $1 \mu \mathrm{g}$ total RNA was used in each microarray experiment, and for amplification and labeling of mRNA the Smart technique (Smart Fluorescent Probe Amplification Kit; BD Clontech, Heidelberg, Germany) was applied according to the manufacturer's protocol. RNA samples from day 1 were labeled with $\mathrm{Cy} 3$, and day 6 or day 21 samples were labeled with $\mathrm{Cy} 5$ ( $\mathrm{Cy}^{\mathrm{TM}} 3$ - and $\mathrm{Cy}^{\mathrm{TM}} 5$-monoreactive dye; Amersham Pharmacia Biotech). Corresponding Cy3and Cy5-labeled samples were mixed, vacuum dried and resuspended in $25 \mu 1$ microarray hybridization buffer (MWGBiotech; Ebersberg, Germany). Prior to hybridization, the samples were heat denaturated at $95^{\circ} \mathrm{C}$ for $5 \mathrm{~min}$. The human 10K (MWG-Biotech) oligo microarray systems on glass slides were used for mRNA profiling. Hybridization of Cy3/Cy5-cDNA was performed using cover slips and a
Table I. Different integrins and their functions.

\begin{tabular}{ll}
\hline Integrin & Receptor for \\
\hline Integrin $\alpha 5 \beta 1$ & Fibronectin \\
Integrin $\alpha 4 \beta 1$ & VCAM, fibronectin \\
Integrin $\alpha 6 \beta 1$ and $\alpha 7 \beta 1$ & Laminin \\
Integrin $\alpha 1 \beta 1$ & Collagen, laminin, tenascin \\
Integrin $\alpha 2 \beta 1$ & Laminin, collagen \\
Integrin $\alpha v 35$ & Osteopontin \\
\hline
\end{tabular}

hybridization chamber for $16 \mathrm{~h}$ at $42^{\circ} \mathrm{C}$ in a water bath. After stringent washing of the glass slides according to the manufacturer's specifications the hybridization signals of the Cy3 and the Cy5 dyes were measured using a microarray laser scanner (GMS418; Affymetrix, MWG-Biotech).

Microarray data analysis and statistics. The ArrayVision (Imaging Research, Inc., St. Catharines, ON, Canada) software was used for evaluation and calculation of signal intensities from the raw data images in 16-bit tagged-image-file (TIF) format as previously described by Bugert et al (30). In brief, for evaluation of hybridization results, we defined a negative $(<3,000)$, a grey area $(3,000-4,999)$ and a positive range $(\geq 5,000)$ of hybridization signal intensities. Signal-tobackground $(\mathrm{S} / \mathrm{B})$ values were calculated by dividing the signal intensity for each spot with the background signal intensities of the hybridized glass slide. Computer-assisted evaluation of the raw data provided the mean signal intensity and the signal-to-background ratio for each individual gene spot. For statistical evaluation the mean signal intensity and standard deviation (SD) were calculated for each spot from the values obtained in the 10 individual experiments. Functional grouping of genes was performed on the basis of the database supplied by the array manufacturer.

Immunohistochemistry. Immunohistochemistry for integrin integrin $\alpha \mathrm{v}$, integrin $\beta 1$, integrin $35, \mathrm{CD} 47$ and the integrinlinked kinase (ILK) was performed by using a streptavidinbiotin complex procedure. Endogenous peroxidase was blocked with $0.3 \%$ hydrogen peroxide for $30 \mathrm{~min}$. Sections were washed with phosphate-buffered saline (PBS) and incubated with normal rabbit serum in PBS for $30 \mathrm{~min}$ at room temperature to block non-specific antibody reaction. The sections were then incubated overnight at $4^{\circ} \mathrm{C}$ with the primary antibody (all from Santa Cruz Biotechnologies, Heidelberg, Germany). The slides were washed by several changes of PBS. The sections were then incubated with a peroxidase-conjugated secondary antibody (Dako, Hamburg, Germany). After being washed twice in PBS, sections were then treated with a streptavidin-biotin-peroxidase complex and peroxidase reaction was performed using diaminobenzidine (DAB) (Dako) as chromogen. The various antibodies were diluted to the desired concentrations in PBS. Controls were carried out by omitting the primary antibody. Light microscopy was performed using a Zeiss Axiophot microscope. 
Table II. Signal intensities of hybridization signals as measured using the microarray laser scanner and calculated by the ArrayVision software in MSC from bone marrow. ${ }^{a}$

\begin{tabular}{|c|c|c|c|c|c|}
\hline Receptor for & Day 0 & Day 20 & Day 0 SD & Day $20 \mathrm{SD}$ & Ratio day $20 /$ day 0 \\
\hline \multicolumn{6}{|l|}{ Fibronectin } \\
\hline Integrin $\alpha 5$ & 11,790 & 9,144 & 4,269 & 4,093 & 0.78 \\
\hline Integrin $\beta 1$ & 32,134 & 15,557 & 3,040 & 3,698 & 0.48 \\
\hline \multicolumn{6}{|c|}{ VCAM, fibronectin } \\
\hline Integrin $\alpha 4$ & 811 & 2,584 & 307 & 821 & 3.19 \\
\hline Integrin $\beta 1$ & 32,134 & 15,557 & 3,040 & 3,698 & 0.48 \\
\hline \multicolumn{6}{|l|}{ Laminin } \\
\hline Integrin $\alpha 6$ & 1,206 & 773 & 241 & 132 & 0.64 \\
\hline Integrin $\alpha 7$ & 2,872 & 4,826 & 1,412 & 2,412 & 1.68 \\
\hline Integrin $\beta 1$ & 32,134 & 15,557 & 3,040 & 3,698 & 0.48 \\
\hline \multicolumn{6}{|c|}{ Collagen, laminin, tenascin } \\
\hline Integrin $\alpha 1$ & 7,345 & 4,639 & 6,079 & 4,269 & 0.63 \\
\hline Integrin $\beta 1$ & 32,134 & 15,557 & 3,040 & 3,698 & 0.48 \\
\hline \multicolumn{6}{|c|}{ Laminin, collagen } \\
\hline Integrin $\alpha 2$ & 23,892 & 30,795 & 9,674 & 3,988 & 1.29 \\
\hline Integrin $\beta 1$ & 32,134 & 15,557 & 3,040 & 3,698 & 0.48 \\
\hline \multicolumn{6}{|l|}{ Osteopontin } \\
\hline Integrin $\alpha \mathrm{v}$ & 4,044 & 3,306 & 1,112 & 1,036 & 0.82 \\
\hline Integrin 35 & 2,958 & 15,601 & 804 & 8,541 & 5.27 \\
\hline \multicolumn{6}{|c|}{ Signaling cascade } \\
\hline CD47 & 1,931 & 2,625 & 1,224 & 1,921 & 1.36 \\
\hline ILK & 22,758 & 23,360 & 11,452 & 11,496 & 1.03 \\
\hline ICAP-1 & 1,041 & 2,147 & 117 & 594 & 2.06 \\
\hline
\end{tabular}

${ }^{\text {aS }}$ ignals were measured on day 1 and after chondrogenic differentiation on day 20. Signals are shown with standard deviation (SD) and the ratio day 20/day 1 .

\section{Results}

Microarray analysis. In MSC from bone marrow (BM-MSC; Table II, Fig. 1), for the components of the fibronectin receptor (integrin $\alpha 5 / \beta 1$ ) a constant expression for integrin $\alpha 5$ (day 0 , 11,790 ; day 20,9,144; ratio day 20/day $0,0.78$ ) was found, and integrin $\beta 1$ was inactivated (day 0, 32,134; day 20, 15,557; ratio day 20/day $0,0.48$ ). The components of the receptor for VCAM and fibronectin (integrin $\alpha 4 / \beta 1$ ) were not expressed (integrin $\alpha 4$ : day 0,811 ; day 20,2,548; ratio day 20/ day $0,3.19$ ) and the gene for integrin $\beta 1$ (day $0,32,134$; day 20, 15,557; ratio day 20/day $0,0.48$ ) was inactivated. The components of the receptor for laminin (integrin $\alpha 6 / \beta 1$ and integrin $\alpha 7 / \beta 1$ ) showed a constant expression of integrin $\alpha 7$ (day 0, 2,872; day 20,4,826; ratio day 20/day 0,1.68) and an inactivation of integrin $\beta 1$ (day $0,32,134$; day 20, 15,557; ratio day 20/day $0,0.64$ ). The components of the receptor for collagen, laminin and tenascin (integrin $\alpha 1 / \beta 1$ ) were inactivated (integrin $\alpha 1$ : day $0,7,345$; day $20,4,639$; ratio day $20 /$ day 0 , 0.63 ; and integrin $\beta 1$ : day $0,32,134$; day $20,15,557$; ratio day $20 /$ day $0,0.48)$. The components of the receptor for laminin and collagen (integrin $\alpha 2 / \beta 1$ ) revealed a constant expression of integrin $\alpha 2$ (day $0,23,892$; day 20,30,795; ratio day 20/day $0,1.29$ ) and an inactivation of integrin $\beta 1$ (day 0, 32,134; day 20, 15,557; ratio day 20/day 0,0.48). For the receptor for osteopontin (integrin $\alpha \mathrm{v} / 35$ ), an inactivation of integrin $\alpha$ v (day 0,4,044; day 20,3,306; ratio day 20/day $0,0.82$ ) and an activation of integrin $\beta 5$ (day $0,2,958$; day $20,15,601$; ratio day 20/day $0,5.27$ ) was found. The components of the intracellular signaling cascade revealed a constant expression of ILK (day 0, 1,931; day 20, 2,625; ratio day 20/day $0,1.36$ ) and of CD47 (day 0, 22,758; day 20, 23,360 ; ratio day $20 /$ day $0,1.03$ ). ICAP- 1 was not expressed (day $0,1,041$; day 20,2,147; ratio day 20/day 0,2.06).

In MSC from adipose tissue (Table III, Fig. 2), the components of the fibronectin receptor (integrin $\alpha 5 / \beta 1$ ) showed an inactivation for integrin $\alpha 5$ (day $0,29,474$; day 20 , 15,615 ; ratio day 20/day $0,0.353$ ), and for integrin $\beta 1$ (day 0 , 7,345 ; day 20, 4,639; ratio day 20/day $0,0.63$ ). The components of the receptor for VCAM and fibronectin (integrin $\alpha 4 / \beta 1$ ) were not expressed (integrin $\alpha 4$ : day $0,1,038$; day 20,1,186; ratio day 20/day $0,1.14$ ) and inactivated (integrin 31 : day 0,7,345; day 20,4,639; ratio day 20/day 0 , 0.63 ). The components of the receptor for laminin (integrin $\alpha 6 / \beta 1$ and integrin $\alpha 7 / \beta 1$ ) revealed no expression of integrin $\alpha 7$ (day 0,1,162; day 20,848; ratio day 20/day 0,0.73) and an inactivation of integrin $\beta 1$ (day $0,7,345$; day 20, 4,639; ratio day 20/day $0,0.63$ ). Integrin $\alpha 6$ (day $0,1,169$; day 20, 
Table III. Signal intensities of hybridization signals as measured using the microarray laser scanner and calculated by the ArrayVision software in MSC from adipose tissue. ${ }^{\mathrm{a}}$

\begin{tabular}{|c|c|c|c|c|c|}
\hline Receptor for & Day 0 & Day 20 & Day 0 SD & Day 20 SD & Ratio day $20 /$ day 0 \\
\hline \multicolumn{6}{|l|}{ Fibronectin } \\
\hline Integrin $\alpha 5$ & 29,474 & 15,615 & 5,283 & 3,389 & 0.53 \\
\hline Integrin $ß 1$ & 7,345 & 4,639 & 6,079 & 4,269 & 0.63 \\
\hline \multicolumn{6}{|c|}{ VCAM, fibronectin } \\
\hline Integrin $\alpha 4$ & 1,038 & 1,186 & 322 & 821 & 1.14 \\
\hline Integrin $\beta 1$ & 7,345 & 4,639 & 6,079 & 4,269 & 0.63 \\
\hline \multicolumn{6}{|l|}{ Laminin } \\
\hline Integrin $\alpha 6$ & 1,169 & 775 & 373 & 54 & 0.66 \\
\hline Integrin $\alpha 7$ & 1,162 & 848 & 399 & 177 & 0.73 \\
\hline Integrin $\beta 1$ & 7,345 & 4,639 & 6,079 & 4,269 & 0.63 \\
\hline \multicolumn{6}{|c|}{ Collagen, laminin, tenascin } \\
\hline Integrin $\alpha 1$ & 824 & 7,498 & 442 & 2,854 & 9.10 \\
\hline Integrin $ß 1$ & 7,345 & 4,639 & 6,079 & 4,269 & 0.63 \\
\hline \multicolumn{6}{|c|}{ Laminin, collagen } \\
\hline Integrin $\alpha 2$ & 5,060 & 11,110 & 2,954 & 4,956 & 2.20 \\
\hline Integrin $\beta 1$ & 7,345 & 4,639 & 6,079 & 4,269 & 0.63 \\
\hline \multicolumn{6}{|l|}{ Osteopontin } \\
\hline Integrin $\alpha v$ & 4,592 & 5,147 & 1,112 & 1,036 & 1.12 \\
\hline Integrin 35 & 8,409 & 15,706 & 4,467 & 2,542 & 1.87 \\
\hline \multicolumn{6}{|c|}{ Signaling cascade } \\
\hline CD47 & 4,745 & 7,618 & 2,586 & 6,470 & 1.61 \\
\hline ILK & 6,468 & 5,532 & 734 & 266 & 0.86 \\
\hline ICAP-1 & 1,041 & 2,147 & 117 & 594 & 2.06 \\
\hline
\end{tabular}

aSignals were measured on day 1 and after chondrogenic differentiation on day 20. Signals are shown with standard deviation (SD) and the ratio day 20/day 1 .

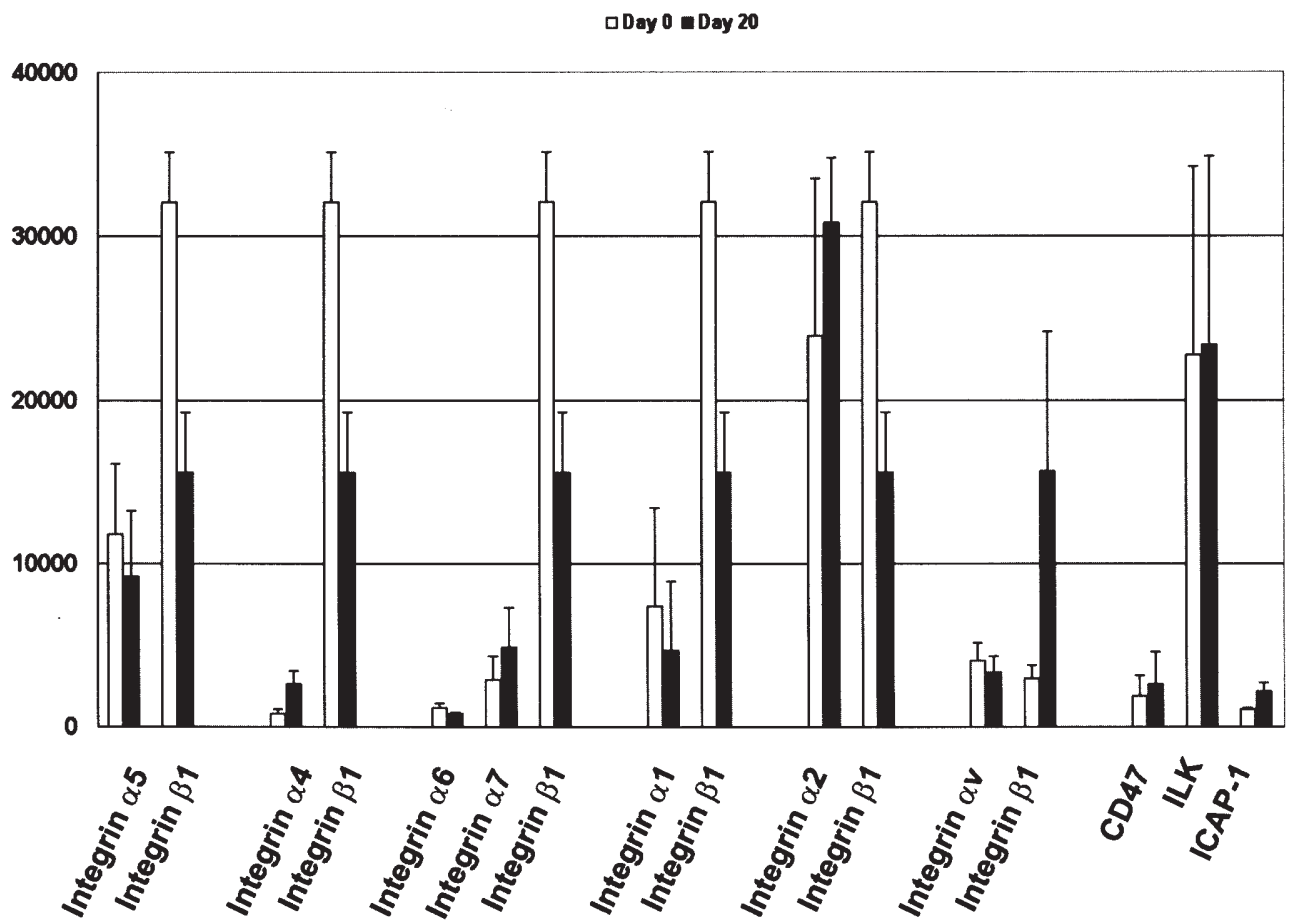

Figure 1. Expression levels of genes for different integrins and integrin-associated proteins in MSC by microarray hybridization analysis. Results from mesenchymal stem cells from bone marrow (BM-MSC) during chondrogenic differentiation for the given genes in undifferentiated MSC (white bars) and chondrocytes differentiated from the MSC (black bars). 


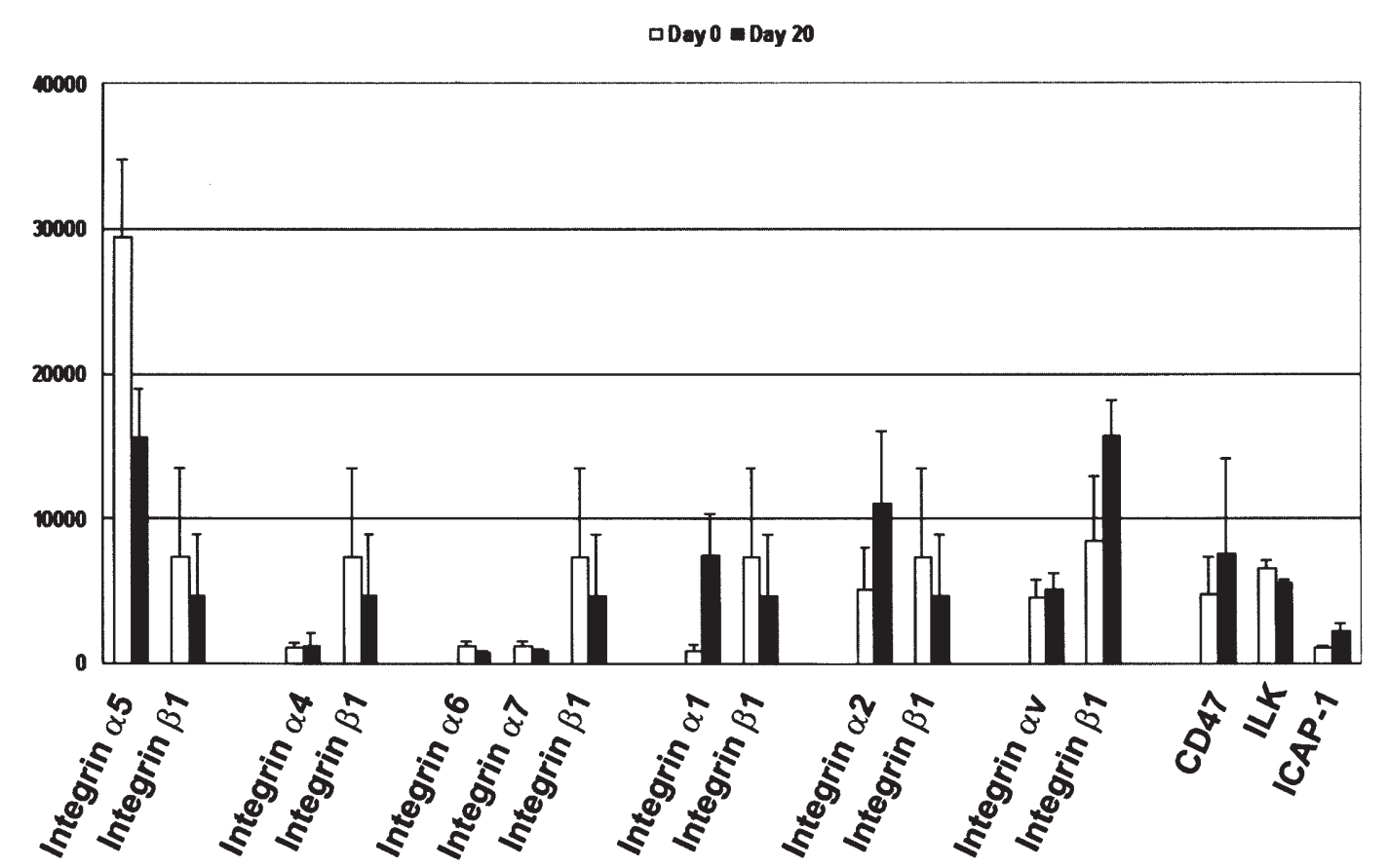

Figure 2. Expression levels of genes for different integrins and integrin-associated proteins in MSC by microarray hybridization analysis. Results from mesenchymal stem cells from adipose tissue (PLA-MSC) during chondrogenic differentiation for the given genes in undifferentiated MSC (white bars) and chondrocytes differentiated from the MSC (black bars).
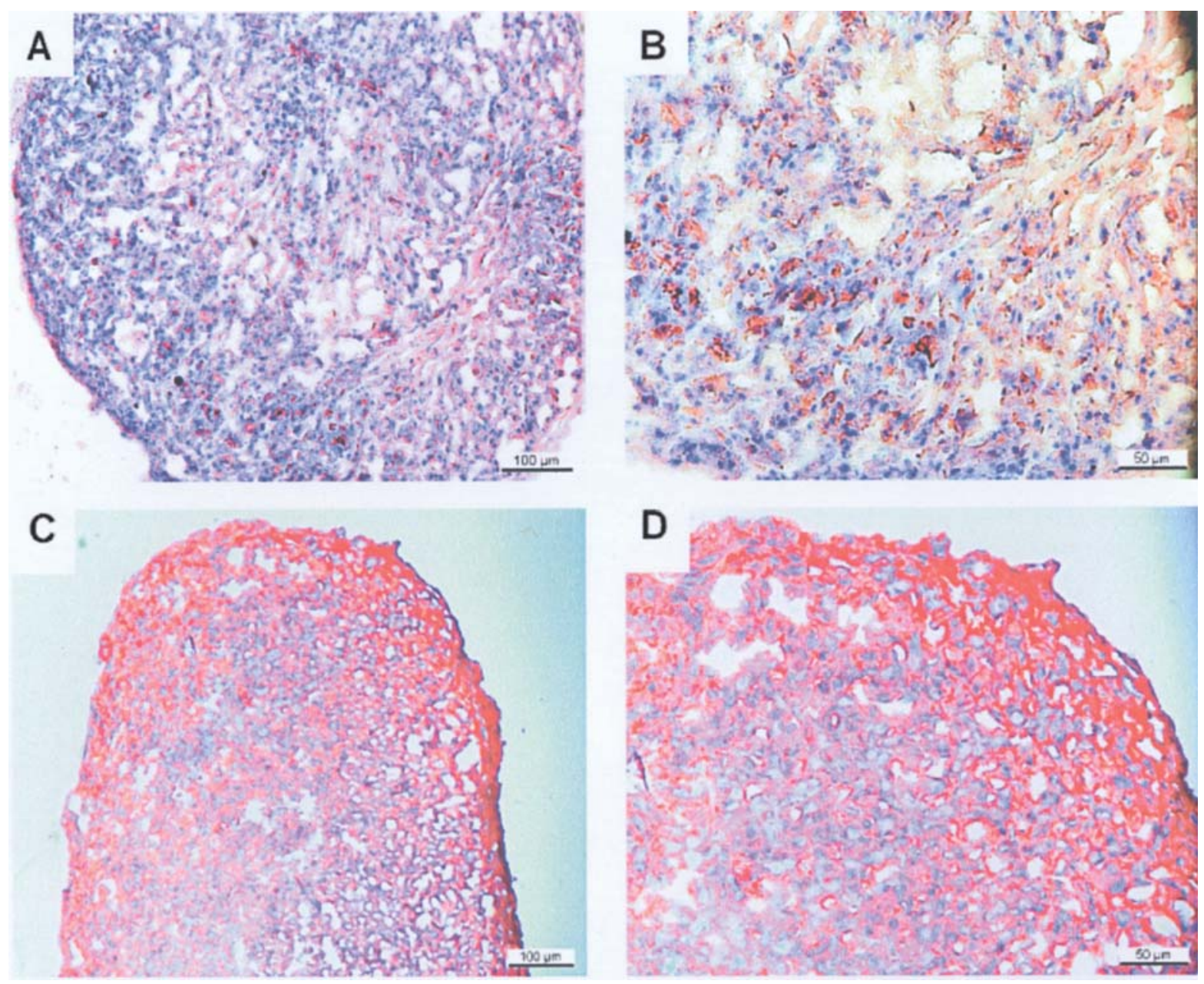

Figure 3. Immunohistochemical staining against different integrins in MSC during chondrogenic differentiation. Day 1 (left) and day 20 (right) of cell culture. (A) Integrin 31 (day 1) in PLA-MSC; (B) integrin B1 (day 20) in PLA-MSC; (C) ILK (day 1) in PLA-MSC; and (D) ILK (day 20) in PLA- MSC. 
Table IV. Immunohistochemical detection of integrins and integrin-associated proteins in freshly isolated MSC (day 1) and during chondrogenic differentiation (days 1, 10, 20 and 30).

\begin{tabular}{|c|c|c|c|c|c|}
\hline \multirow{2}{*}{$\begin{array}{l}\text { Antibody specific } \\
\text { for BM-MSC }\end{array}$} & \multicolumn{5}{|c|}{ Staining pattern ${ }^{\mathrm{a}}$} \\
\hline & Day 0 & Day 1 & Day 10 & Day 20 & Day 30 \\
\hline Integrin $\alpha \mathrm{V}$ & +++ & + & ++ & +++ & ++ \\
\hline Integrin $\beta 5$ & ++ & + & +++ & +++ & ++ \\
\hline Integrin $ß 1$ & ++ & + & ++ & ++ & + \\
\hline CD47 & +++ & ++ & +++ & +++ & ++ \\
\hline ILK & +++ & +++ & +++ & ++ & ++ \\
\hline \multirow{2}{*}{$\begin{array}{l}\text { Antibody specific } \\
\text { for PLA-MSC }\end{array}$} & \multicolumn{5}{|c|}{ Staining pattern ${ }^{a}$} \\
\hline & Day 0 & Day 1 & Day 10 & Day 20 & Day 30 \\
\hline Integrin $\alpha \mathrm{V}$ & +++ & ++ & ++ & ++ & ++ \\
\hline Integrin $B 5$ & +++ & +++ & ++ & ++ & ++ \\
\hline Integrin $ß 1$ & ++ & ++ & ++ & ++ & ++ \\
\hline CD47 & +++ & ++ & ++ & +++ & +++ \\
\hline ILK & ++ & ++ & +++ & +++ & +++ \\
\hline
\end{tabular}

${ }^{a}$ The amount of cells stained by the monoclonal antibodies is symbolized by $++++(80-100 \%),+++(60-70 \%),++-+++(50-60 \%),++(40-50 \%)$, $+-++(30-40 \%),+(20-30 \%), \pm(<20 \%)$, and - no staining.

775 ; ratio day $20 /$ day $0,0.66)$ was not expressed. As for the receptor for collagen, laminin and tenascin (integrin $\alpha 1 / \beta 1$ ), a strong activation of integrin $\alpha 1$ (day 0, 824; day 20, 7,498; ratio day $20 /$ day $0,9.1$ ) and an inactivation of integrin $\beta 1$ (day 0,7,345; day 20,4,639; ratio day 20/day 0,0.63) was found. The receptor for laminin and collagen (integrin $\alpha 2 / \beta 1$ ) revealed an activation of integrin $\alpha 2$ (day $0,5,060$; day 20, 11,110 ; ratio day $20 /$ day $0,2.20$ ) and an inactivation of integrin $\beta 1$ (day $0,7,345$; day 20,4,639; ratio day 20/day 0 , 0.63 ). For the receptor for osteopontin (integrin $\alpha v / 35$ ), a constant expression of integrin $\alpha \mathrm{v}$ (day $0,4,592$; day 20 , 5,147 ; ratio day 20/day $0,1.12$ ) and an activation of integrin 35 (day $0,8,409$; day 20, 15,706; ratio day 20/day $0,1.87$ ) was found. The components of the intracellular signaling cascade showed a constant expression of ILK (day 0, 6,488; day 20, 5,532; ratio day 20/day $0,0.86$ ) and CD47 (day 0, 4,745; day 20,7,618; ratio day 20/day $0,1.61$ ). ICAP-1 was not expressed (day 0, 1,041; day 20,2,147; ratio day 20/day 0 , 2.06).

Immunohistochemistry. The analysis of integrin expression at the protein level was analysed with monoclonal antibodies against integrin $\alpha \mathrm{v}$, integrin $\beta 1$, integrin $35, \mathrm{CD} 47$ and the integrin-linked kinase (ILK). During the entire process of chondrogenic differentiation, a constant expression for all markers was found (Fig. 3, Table IV).

\section{Discussion}

The field of regenerative medicine encompasses various areas of technology, such as tissue engineering, stem cells, and cloning. Tissue engineering, one of the major components of regenerative medicine, follows the principles of cell transplantation, materials science, and engineering towards the development of biological substitutes that can restore and maintain normal function.

Bone marrow-derived stem cells have been studied for decades, and are well characterized and safe even in clinical settings. For clinical applications of stem cell transplantation therapy, direct manipulation of cells and their interactions would be desirable. Hitherto, establishing distinct protocols for precisely inducing and maintaining cellular differentiation with a defined phenotype and function has been extremely challenging. In addition, the elucidation of cell-cell and cellmatrix interactions is necessary, and the integrins as a family of adhesion receptors mediating these stimuli are a promising target for research.

As mentioned above the harvesting of BM is a highly invasive procedure and the number, differentiation potential, and maximal life span of MSC from BM decline with increasing age $(11,12)$. Therefore, alternative sources from which to isolate MSC are subject to intensive investigation. Adipose tissue (AT) is an alternative source that can be obtained by a less invasive method and in larger quantities than BM.

Expression of integrins was analysed in MSC from bone marrow and adipose tissue during chondrogenic differentiation. The components of the fibronectin receptor (integrin $\alpha 5 / \beta 1$ ) demonstrated a diminished expression with ongoing differentiation in both types of MSC at the RNA and protein level alike.

With RT-PCR analysis it was previously shown that freshly isolated MSC express collagen 2 and 10 (31). These previous results agree with the finding of high expression of 
integrin $\alpha 5 \beta 1$ in undifferentiated MSC, as the interaction of MSC and components of the ECM (e.g. collagen 2) via this receptor are known. Thus, integrin $\alpha 5 \beta 1$ may exert an influence on the cellular phenotype in undifferentiated MSC, and with ongoing differentiation this receptor seems to become less important in both types of MSC at the same time.

The receptor for VCAM and fibronectin (integrin $\alpha 4 / 31$ ) was not expressed by MSC from BM and AT in terms of integrin $\alpha 4$ and was inactivated during chondrogenic differentiation for integrin $\beta 1$, respectively. This receptor does not seem to be involved in signaling during chondrogenic differentiation.

The components of the receptor for laminin (integrin $\alpha 6 / \beta 1$ and $\alpha 7 / \beta 1$ ) showed no expression of integrin $\alpha 6$ and $\alpha 7$ in the two types of MSC as well as a diminished expression of integrin $B 1$ during chondrogenic differentiation. Thus, this receptor does not appear to play an important role during chondrogenic differentiation.

The receptor for collagen, laminin and tenascin (integrin $\alpha 1 / \beta 1)$ revealed a strong activation of integrin $\alpha 1$ in both stem cell types, whereas integrin $\beta 1$ showed a diminished expression with ongoing differentiation. These results do not allow a specific conclusion to be made concerning the role of this receptor during chondrogenic differentiation.

The osteopontin receptor (integrin $\alpha \mathrm{v} / 35$ ) showed constant expression for integrin $\alpha \mathrm{v}$ in both types of MSC. Integrin $B 5$ was inactivated in BM-MSC and was activated in PLA-MSC. It has been established that integrin 35 plays a role in binding vitronectin (32). In addition, it has been suggested that the osteopontin receptor might be involved in processes of cellular migration and proliferation, especially in smooth muscle cells during vascular trauma (33). In addition, this receptor may assist in cellular differentiation in vitro (34). The expression of this receptor during chondrogenic differentiation might reflect its influence during the generation of a distinct ECM. The adhesion to surrounding ECM molcules may guide the process of differentiation.

The analysis of the components of the receptors for laminin and collagen (integrin $\alpha 2 / \beta 1$ ) resulted in an inactivation of integrin $\alpha 2$ in BM-MSC, and a rising expression during chondrogenic differentiation in PLA-MSC. As mentioned above, integrin $B 1$ showed diminishing expression during chondrogenic differentiation. For this receptor, a specific role during chondrogenic differentiation was not established. Yet one possible role may include its adhering to collagens to facilitate the synthesis of ECM. This process is not able to be analysed in a monolayer culture.

In summary, after comparing the expression patterns in the two stem cell types, we found different expression patterns only at the RNA level for two receptors, suggesting that there are no significant differences in chondrogenic differentiation capacity and the expression of integrins. Establishing new sources for MSC may have a high impact on the clinical usage of these cells. Exploitation may be related to the abundance and expansion capacity of MSC. Based on our results, both $\mathrm{BM}$ and AT are reliable sources for isolating and propagating MSC in autologous settings. The advantages of PLA-MSC include the easy harvesting and handling. One argument against using AT is the limited availability in certain patients.
However, we believe that due to the high quantities of ATMSC, small fat reservoirs are also sufficient for MSC isolation. For the past decades, BM has been utilized as the main source of MSC for clinical applications, such as the treatment of osteogenesis imperfecta, graft versus host disease, or acute myocardial infarction (35-37). As BM-MSC demonstrate an age-dependent decrease in number, frequency, and differentiation capacity, they may be clinically inefficient when derived from elderly patients. Taking into account all of these factors, PLA-MSC may provide a reliable first source in reference to their abundance, easy harvest, and high MSC frequency.

In the present study, we analysed the expression patterns of integrins and integrin-related signaling proteins. One of the candidates for signal transmission is the fibronectin receptor which may play a role in freshly isolated cells. Other receptors, e.g. for collagen, laminin and tenascin, do not appear to be involved in signal transduction. The receptor for osteopontin seems to play a role during chondrogenic differentiation. In addition, the receptor for laminin and collagen may assist the beginning of chondrogenic differentiation. Intracellularly, ILK and CD47, but not ICAP-1, may be involved in transduction of the integrin-dependent signals.

Integrin-mediated signaling seems to play an important role in the generation and maintenance of the chondrocytic phenotype during chondrogenic differentiation. To fully harness the potential of these cells, future studies should be directed to ascertain their cellular and molecular characteristics for optimal identification, isolation, and expansion.

\section{References}

1. Atala A: Tissue engineering and regenerative medicine: concepts for clinical application. Rejuvenation Res 7: 15-31, 2004.

2. Atala A: Recent developments in tissue engineering and regenerative medicine. Curr Opin Pediatr 18: 167-171, 2006.

3. Goessler UR, Hormann K and Riedel F: Tissue engineering with chondrocytes and function of the extracellular matrix (Review). Int J Mol Med 13: 505-513, 2004.

4. Oakes BW: Orthopaedic tissue engineering: from laboratory to the clinic. Med J Aust 180: S35-S38, 2004.

5. Caplan AI: Mesenchymal stem cells. J Orthop Res 9: 641-650, 1991.

6. Gao J and Caplan AI: Mesenchymal stem cells and tissue engineering for orthopaedic surgery. Chir Organi Mov 88: 305-316, 2003.

7. Johnstone B, Hering TM, Caplan AI, Goldberg VM and Yoo JU: In vitro chondrogenesis of bone marrow-derived mesenchymal progenitor cells. Exp Cell Res 238: 265-272, 1998.

8. Ringe J, Kaps C, Burmester GR and Sittinger M: Stem cells for regenerative medicine: advances in the engineering of tissues and organs. Naturwissenschaften 89: 338-351, 2002.

9. Pei X: Stem cell engineering: the new generation of cellular therapeutics. Int J Hematol 76 (suppl 1): 155-156, 2002.

10. Beyer Nardi $\mathrm{N}$ and da Silva Meirelles L: Mesenchymal stem cells: isolation, in vitro expansion and characterization. Handb Exp Pharmacol: 249-282, 2006.

11. Nishida S, Endo N, Yamagiwa H, Tanizawa T and Takahashi HE: Number of osteoprogenitor cells in human bone marrow markedly decreases after skeletal maturation. J Bone Miner Metab 17: 171-177, 1999

12. Mueller SM and Glowacki J: Age-related decline in the osteogenic potential of human bone marrow cells cultured in threedimensional collagen sponges. J Cell Biochem 82: 583-590, 2001.

13. Stenderup K, Justesen J, Clausen C and Kassem M: Aging is associated with decreased maximal life span and accelerated senescence of bone marrow stromal cells. Bone 33: 919-926, 2003. 
14. Zuk PA, Zhu M, Ashjian P, et al: Human adipose tissue is a source of multipotent stem cells. Mol Biol Cell 13: 4279-4295, 2002.

15. Zuk PA, Zhu M, Mizuno H, et al: Multilineage cells from human adipose tissue: implications for cell-based therapies. Tissue Eng 7: 211-228, 2001.

16. Loeser RF, Carlson CS and McGee MP: Expression of beta 1 integrins by cultured articular chondrocytes and in osteoarthritic cartilage. Exp Cell Res 217: 248-257, 1995.

17. Goessler UR, Bieback K, Bugert $\mathrm{P}$, et al: In vitro analysis of integrin expression during chondrogenic differentiation of mesenchymal stem cells and chondrocytes upon dedifferentiation in cell culture. Int J Mol Med 17: 301-307, 2006.

18. Goessler UR, Bugert P, Bieback K, et al: Differential modulation of integrin expression in chondrocytes during expansion for tissue engineering. In Vivo 19: 501-507, 2005.

19. Goessler UR, Hormann K and Riedel F: Tissue engineering with adult stem cells in reconstructive surgery (Review). Int J Mol Med 15: 899-905, 2005.

20. Hering TM: Regulation of chondrocyte gene expression. Front Biosci 4: D743-D761, 1999.

21. Hynes RO: Integrins: versatility, modulation, and signaling in cell adhesion. Cell 69: 11-25, 1992.

22. Giancotti FG and Ruoslahti E: Integrin signaling. Science 285: 1028-1032, 1999

23. Albelda SM and Buck CA: Integrins and other cell adhesion molecules. FASEB J 4: 2868-2880, 1990.

24. Salter DM, Hughes DE, Simpson R and Gardner DL: Integrin expression by human articular chondrocytes. Br J Rheumatol 31: 231-234, 1992.

25. Lee JW, Qi WN and Scully SP: The involvement of beta1 integrin in the modulation by collagen of chondrocyte-response to transforming growth factor-beta1. J Orthop Res 20: 66-75, 2002.

26. Hannigan GE, Leung-Hagesteijn C, Fitz-Gibbon L, et al: Regulation of cell adhesion and anchorage-dependent growth by a new beta 1-integrin-linked protein kinase. Nature 379: 91-96, 1996.

27. Bouvard D and Block MR: Calcium/calmodulin-dependent protein kinase II controls integrin alpha5beta1-mediated cell adhesion through the integrin cytoplasmic domain associated protein-1alpha. Biochem Biophys Res Commun 252: 46-50, 1998.
28. Mawby WJ, Holmes CH, Anstee DJ, Spring FA and Tanner MJ: Isolation and characterization of CD47 glycoprotein: a multispanning membrane protein which is the same as integrinassociated protein (IAP) and the ovarian tumour marker OA3. Biochem J 304: 525-530, 1994.

29. Mackay AM, Beck SC, Murphy JM, Barry FP, Chichester CO and Pittenger MF: Chondrogenic differentiation of cultured human mesenchymal stem cells from marrow. Tissue Eng 4: 415-428, 1998.

30. Bugert P, Dugrillon A, Gunaydin A, Eichler H and Kluter H: Messenger RNA profiling of human platelets by microarray hybridization. Thromb Haemost 90: 738-748, 2003.

31. Wang WG, Lou SQ, Ju XD, Xia K and Xia JH: In vitro chondrogenesis of human bone marrow-derived mesenchymal progenitor cells in monolayer culture: activation by transfection with TGF-beta2. Tissue Cell 35: 69-77, 2003.

32. McLean JW, Vestal DJ, Cheresh DA and Bodary SC: cDNA sequence of the human integrin beta 5 subunit. J Biol Chem 265: 17126-17131, 1990.

33. Liaw L, Almeida M, Hart CE, Schwartz SM and Giachelli CM: Osteopontin promotes vascular cell adhesion and spreading and is chemotactic for smooth muscle cells in vitro. Circ Res 74: 214-224, 1994.

34. Gladson CL, Dennis C, Rotolo TC, Kelly DR and Grammer JR: Vitronectin expression in differentiating neuroblastic tumors: integrin alpha $\mathrm{v}$ beta 5 mediates vitronectin-dependent adhesion of retinoic-acid-differentiated neuroblastoma cells. Am J Pathol 150: 1631-1646, 1997.

35. Horwitz EM, Gordon PL, Koo WK, et al: Isolated allogeneic bone marrow-derived mesenchymal cells engraft and stimulate growth in children with osteogenesis imperfecta: Implications for cell therapy of bone. Proc Natl Acad Sci USA 99: 8932-8937, 2002.

36. Le Blanc K, Rasmusson I, Sundberg B, et al: Treatment of severe acute graft-versus-host disease with third party haploidentical mesenchymal stem cells. Lancet 363: 1439-1441, 2004.

37. Chen SL, Fang WW, Qian J, et al: Improvement of cardiac function after transplantation of autologous bone marrow mesenchymal stem cells in patients with acute myocardial infarction. Chin Med J 117: 1443-1448, 2004. 